\title{
Wireless Sensor Networks with RF Energy Harvesting: Energy Models and Analysis
}

\author{
M. Yousof Naderi, Kaushik R. Chowdhury, Stefano Basagni \\ Department of Electrical and Computer Engineering, Northeastern University, Boston, MA, U.S.A \\ E-mail: \{naderi, krc, basagni\}@ece.neu.edu
}

\begin{abstract}
This paper formulates the location-dependent power harvesting rates in generalized $2 \mathrm{D}$ and $3 \mathrm{D}$ placement of multiple Radio Frequency (RF) Energy Transmitters (ETs) for recharging the nodes of a wireless sensor network (WSN). In particular, we study the distributions of total available and harvested power over the entire WSN. We provide closed matrix forms of harvestable power at any given point in space due to the action of concurrent energy transfer from multiple ETs, explicitly considering constructive and destructive interference of the transmitted energy signals. We also analyze the performance of energy transfer in the WSN through power outage probability, interference, and harvested voltage as a function of the wireless power received from the ETs. Our results reveal that the network wide received power and interference power from concurrent energy transfers exhibit Log-Normal distributions, and the harvested voltage over the network follows a Rayleigh distribution.
\end{abstract}

\section{INTRODUCTION}

Electromagnetic waves carry energy in the form of electric and magnetic fields, which can be converted (with some losses) and stored as energy at the receiving front-end, and used to power the processing and communication circuits of the nodes of a wireless sensor network (WSN). The ability of transferring energy via contact-less radio frequency (RF) will ensure the sensor nodes to remain operational for long times, without the need of costly battery replacement efforts [1]. Current prototypes of RF transfer, however, have limited charging range (few meters) and efficiency (40 to $60 \%$ ). This imposes the concurrent and coordinated use of multiple ETs to power an entire WSN [2].

While multiple ETs are needed to ensure high energy transfer rates, they introduce interference among RF waves from different ETs, leading to significant and various constructive and destructive combinations over the network deployment area. Being able to compute the energy harvestable at a given point in space is a non-trivial task, as it depends on the relative locations of active ETs, path loss information, and on the different distances from the ETs and a receiver at that point. However, estimating the harvestable energy is a key first step in network planning and protocol design for long-lived WSNs. For example, at the link layer, sensors placed in regions that will receive energy at low transfer rates should have lower duty cycles. At the network layer, routes can be pre-computed to pass through regions with high energy transfers. Task assignment, at the application layer can also be facilitate by knowing how much energy will be available at a node at a certain time.
Deriving closed form expressions of the energy harvestable at any point in the deployment space of the WSN is the main aim and contribution of this paper.

Our approach starts from considering the path-loss models between one transmitter and one receiver, such as the one expressed by the Friis transmission equation [3], [4] estimating the received power between two nodes. We then incorporate in these models results from array factor calculation in an N-element antenna array and phased array [5], [6], [7] used to estimate the cumulative contribution of all the isotropic radiating elements at any far-field point. Hardware design factors, such as the diode operational parameters used in voltage multiplier sections of the energy harvesting circuit and RF-to-DC conversion efficiency, are integrated in our communication-centric analytical models. The resulting closed matrix form expressions give an estimate of the harvestable energy from multiple wireless ETs at any point, explicitly considering the unique features of constructive and destructive combination of RF waves. Our analytical approach first concentrates on the case of two and $N$ ETs in the plane, and then generalizes the results to $N$ ETs in 3D. Through our derived models we analyze and determine optimal distributions of power outage, energy interference, and harvested voltage and power over the whole WSN. Our analysis shows that the received power from multiple ETs over the entire network and the network energy interference caused by concurrent RF energy transfer has Log-Normal distributions. In addition, the harvested voltage over the whole WSN has Raleigh distribution due to use of either diodes or transistors, which are typically used in energy harvesting circuits, and to their input powervoltage curves.

The rest of paper is organized as follows. In Section II we determine the harvestable energy in the plane. Section III extends generalize our findings to 3D spaces. In Section IV we study the impact of wireless energy interference from multiple ETs over the entire WSN, and present the observed distributions of energy-related metrics. Finally, Section V concludes the paper.

\section{2D RF ENERGY MODEL}

In this section we develop analytical expressions for the total harvestable energy from two ETs at any given location in the plane. We then extend these expressions to the case of $N$ ETs in the plane. The ETs and sensors are assumed 


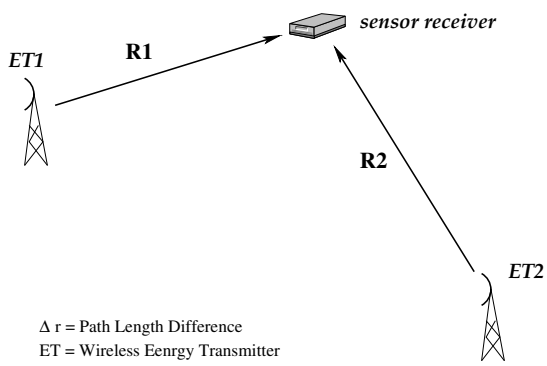

Fig. 1: Two ETs transferring power on the same frequency to a receiver node at distance $R_{1}$ and $R_{2}$.

to be equipped with omnidirectional dipole antennas, and ETs transfer RF waves with the same initial phase. The RF waves carry energy in the form of the electric field. Computation of the intensity of harvestable power from two ETs starts with computing the sum of their electric fields at any given location. The electric field $(E)$ from an ET measured by a receiver device at distance $R$ is:

$$
E=\sqrt{Z_{0} S} e^{-j k R}=\sqrt{Z_{0} S} e^{-j\left(\frac{2 \pi}{\lambda}\right) R},
$$

where $Z_{0}$ is a physical constant indicates the waveimpedance of a plane wave in free space [8]. Moreover, $S$ is the power spatial density at distance $R$ (i.e., the power per unit area), and $k$ is the wavenumber of the energy wave (i.e., the magnitude of the energy wave vector). The term $k R$ indicates the phase shift of the transmitted energy signal at distance $R$. Here, $S=\frac{G_{t} P_{t}}{4 \pi R^{2}}$, where $G_{t}$ is the transmitter gain and $P_{t}$ is the output power of the ET. Fig. 1 shows a scenario with two ETs.

The total electric field $E_{T}$ at the receiver when both ETs are transmitting energy is merely the superposition of their individual electric fields:

$$
E_{T}=E_{1}+E_{2}=\sqrt{Z_{0} S_{1}} e^{-j k R_{1}}+\sqrt{Z_{0} S_{2}} e^{-j k R_{2}},
$$

where the first term is the electric field from $E T_{1}$ and the second term is the electric field from $E T_{2}$. The magnitude of the field can be expressed as:

$$
\left.\left|E_{T}\right|=\sqrt{\left|E_{1}\right|^{2}+\left|E_{2}\right|^{2}+2\left|E_{1}\right|\left|E_{2}\right| \cos \left(k\left(R_{2}-R_{1}\right)\right.}\right) .
$$

Therefore, the density of the total transferred power at the receiver is:

$$
S_{T}=\frac{\left|E_{T}\right|^{2}}{Z_{0}}=S_{1}+S_{2}+2 \sqrt{S_{1} S_{2}} \cos (k \Delta r),
$$

where $\Delta r=\left|R_{1}-R_{2}\right|$ is the difference of the distances between the two ETs and the receiver. Since an ET is an isotropic radiator (an EM source radiating the same power in all directions), the expression for the magnitude of $E$ can be expanded as follows:

$$
S_{T}=\frac{G_{1} P_{1}}{4 \pi R_{1}^{2}}+\frac{G_{2} P_{2}}{4 \pi R_{2}^{2}}+\frac{2}{4 \pi} \sqrt{\frac{P_{1} P_{2} G_{1} G_{2}}{R_{1}^{2} R_{2}^{2}}} \cos (k \Delta r),
$$

where $P_{1}$ and $P_{2}$ are the transmission powers and $G_{1}$ and $G_{2}$ are the transmission gains of $E T_{1}$ and $E T_{2}$, respectively.

The aperture of the antenna of the receiver determines how well it can harvest power from an incoming EM wave. The total available power at an isotropic receiving antenna with an effective area $\mathrm{A}$ is given by:

$$
P_{T}^{r}=S_{T} A=S_{T} G_{r}\left(\frac{\lambda^{2}}{4 \pi}\right),
$$

where $G_{r}$ is the gain of the RF harvester antenna. The total received power from two ETs would be as follows:

$$
\begin{aligned}
& P_{T}^{r}=G_{1} P_{1} G_{r}\left(\frac{\lambda}{4 \pi R_{1}}\right)^{2}+G_{2} P_{2} G_{r}\left(\frac{\lambda}{4 \pi R_{2}}\right)^{2} \\
& \quad+2\left(\frac{\lambda}{4 \pi \sqrt{R_{1} R_{2}}}\right)^{2} G_{r} \sqrt{G_{1} G_{2}} \sqrt{P_{1} P_{2}} \cos (k \Delta r) .
\end{aligned}
$$

If two energy transmitters have the same antenna gain (i.e., $G_{t}$ ) and transmission power (i.e., $P_{t}$ ), then the total received energy at the receiver node simplifies to:

$$
P_{T}^{r}=P_{t} G_{t} G_{r}\left(\frac{\lambda}{4 \pi}\right)^{2}\left(\frac{1}{R_{1}^{2}}+\frac{1}{R_{2}^{2}}+\frac{2 \cos (k \Delta r)}{R_{1} R_{2}}\right) .
$$

According to the RF wireless charging model, the amount of harvested power by the RF energy harvesting receiver would be

$$
P_{H}=\eta P_{T}^{r},
$$

where $\eta$ is the RF-to-DC conversion efficiency. This formula captures both destructive and constructive interferences at any given location by considering the path lengths as well as the path differences between the ETs and the receiver. The harvested voltage could be found by $V_{H}=F\left(P_{T}^{r}\right)$ where the function $F$ relates input power to harvested voltage and depends on the energy harvesting circuit [9].

Similarly, we can obtain the total received power from $N$ ETs as follows:

$$
\begin{aligned}
P_{T}^{r}= & G_{r}\left(\frac{\lambda}{4 \pi}\right)^{2}\left[\sum_{i=1}^{N} \frac{P_{i} G_{i}}{R_{i}^{2}}+\right. \\
& \left.\sum_{\substack{i=1 \\
i \neq j}}^{N} \sum_{j=1}^{N} \frac{\sqrt{G_{i} G_{j} P_{i} P_{j}}}{R_{i} R_{j}} \cos \left(k\left(\Delta r_{i j}\right)\right)\right],
\end{aligned}
$$

where $P_{i}$ and $G_{i}$ are the transmission power and the transmission gain of $E T_{i}$, respectively, and $G_{r}$ is the gain of the receiver antenna. If all ETs have the same antenna gain and transmission powers, then the total received energy at the receiver node simplifies to:

$$
P_{T}^{r}=P_{t} G_{t} G_{r}\left(\frac{\lambda}{4 \pi}\right)^{2}\left[\sum_{i=1}^{N} \frac{1}{R_{i}^{2}}+\sum_{\substack{i=1 \\ i \neq j}}^{N} \sum_{j=1}^{N} \frac{\cos \left(k\left(\Delta r_{i j}\right)\right)}{R_{i} R_{j}}\right],
$$

which in matrix form can be written as follows:

$$
P_{T}^{r}=(P)(\operatorname{corr})\left(R^{-1}\right)
$$




$$
\operatorname{corr}=\left[\begin{array}{cccc}
1 / R_{1} & \cos \left(k\left(R_{1}-R_{2}\right)\right) / R_{1} & \ldots & \cos \left(k\left(R_{1}-R_{n}\right)\right) / R_{1} \\
\cos \left(k\left(R_{2}-R_{1}\right)\right) / R_{2} & 1 / R_{2} & & \\
\vdots & \vdots & \ddots & \\
\cos \left(k\left(R_{n}-R_{1}\right)\right) / R_{n} & \ldots & & 1 / R_{n}
\end{array}\right]
$$

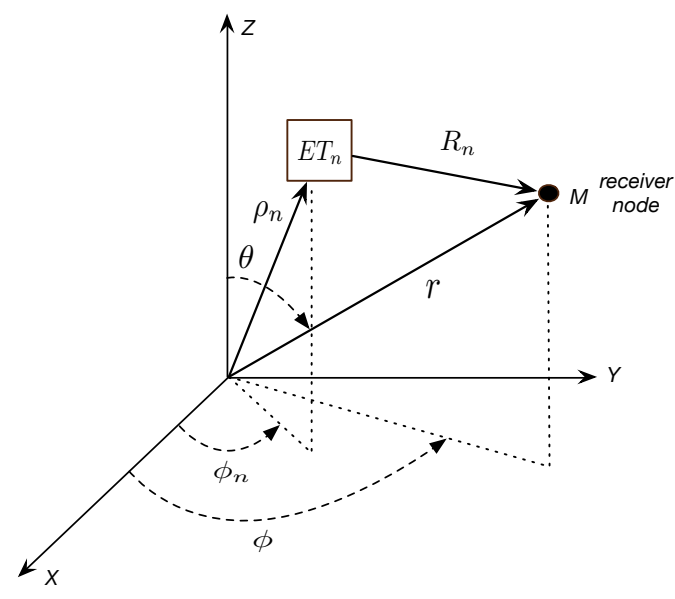

Fig. 2: Geometrical parameters of an $E T_{n}$ transferring wireless energy to a receiver at far-field point $M$.

where $P$ is a constant row vector with size $N$ defined as $\left(P_{t} G_{t} G_{r}\left(\frac{\lambda}{4 \pi}\right)^{2}\right) \cdot \mathbb{1}^{T}$, the $N \times N$ correlation matrix corr is shown in Equation (1), and the distance matrix $R^{-1}$ is:

$$
R^{-1}=\left[\begin{array}{c}
1 / R_{1} \\
1 / R_{2} \\
\vdots \\
1 / R_{n}
\end{array}\right]
$$

\section{3D RF ENERGY MODEL}

In practice and for most applications of energy harvesting sensor network such as health-care, structural health monitoring, smart home, and so forth, we need to deal with threedimensions, where the heights of ETs and sensor nodes are not necessary the same. Accordingly, in this section we first we explain the coordinate system for computing transmitted power from an ET, and then we derive the analytical model of harvestable energy for three-dimensional case of a sensor network with $N$ wireless energy transmitters.

Fig. 2 shows the geometrical parameters for finding the electric field of $E T_{n}$ with respect to the phase reference at origin. We assume the receiver is located at a given point $\mathrm{M},(r, \phi, \theta)$, and it is a far-field point in regard to all energy transmitters. Here, $\overrightarrow{\rho_{n}}$ is the vector from the origin to the $E T_{n}$ (ET vector); $\vec{r}$ is the vector from origin to the observation point (energy transfer direction vector), and $\overrightarrow{R_{n}}$ is the distance vector from the ET to the receiver. Moreover, using spherical coordinates, $x=r \sin (\theta) \cos (\phi)$, $y=r \sin (\theta) \sin (\phi)$, and $z=r \cos (\theta)$, respectively. For $E T_{n}$, the electric field at a far-field point, $\mathrm{M}$, can be expressed as

$$
E_{n}=\sqrt{Z_{0} S_{n}} e^{-j k\left|\overrightarrow{R_{n}}\right|}=\sqrt{Z_{0} S_{n}} e^{-j \gamma_{n}}
$$

where $k=\frac{2 \pi}{\lambda}$ is the wave number, and $\gamma_{n}$ is the phase shift of emitted energy signal from $E T_{n}$ at the receiver with respect to the origin, and $S_{n}$ is the power density of transmitted signal at point $\mathrm{M}$ and would be computed as

$$
S_{n}=\frac{G_{n} P_{n}}{4 \pi\left|\overrightarrow{R_{n}}\right|^{2}} \sin ^{2}(\theta)
$$

where $G_{n}$ ) is the gain of $E T_{n}$ assuming its antenna is oriented to $\hat{z}$ direction and $P_{n}$ is the transmission power. Using spherical coordinates, $R_{n}$ for a far-field point $\mathrm{M}$ (i.e. $r \gg \rho_{n}$ ) can be approximated as follows:

$$
\begin{gathered}
\overrightarrow{R_{n}}=\vec{r}-\overrightarrow{\rho_{n}} \\
\left|\overrightarrow{R_{n}}\right|=|\vec{r}|-\frac{\vec{r} \cdot \overrightarrow{\rho_{n}}}{|\vec{r}|} \\
\left|\overrightarrow{R_{n}}\right|=|\vec{r}|-\left|\overrightarrow{\rho_{n}}\right| \sin (\theta) \cos \left(\phi-\phi_{n}\right)
\end{gathered}
$$

Thus, the electric field of $E T_{n}$ at the receiver located in point $\mathrm{M}$ would be found as

$$
E_{n}=\sin (\theta) \sqrt{\frac{Z_{0} G_{n} P_{n}}{4 \pi R_{n}^{2}}}\left(e^{-j k r} e^{j k \rho_{n} \sin (\theta) \cos \left(\phi-\phi_{n}\right)}\right)
$$

We assume the location of $N$ energy transmitters are given by $\overrightarrow{\rho_{1}}, \overrightarrow{\rho_{2}}, \overrightarrow{\rho_{3}}, \ldots ., \overrightarrow{\rho_{n}}$. We need to find the received and harvestable power at point $\mathrm{M}$, determined by $(r, \phi, \theta)$. Similar to the 2D case, the total electric field strength at the receiver, when $N$ ETs are transmitting simultaneously, is the superposition of individual received electric fields. Hence, the density of total transferred power at the receiver can be calculated as follows,

$S_{T}=\frac{\left|E_{T}\right|^{2}}{Z_{0}}=\frac{1}{Z_{0}}\left[\sum_{n=1}^{N}\left|E_{n}\right|^{2}+\sum_{\substack{i=1 \\ i \neq j}}^{N} \sum_{j=1}^{N}\left(\left|E_{i}\right|\left|E_{j}\right| \cos \left(\gamma_{i}-\gamma_{j}\right)\right)\right]$,

where the relative phase difference of EM waves from $E T_{i}$ and $E T_{j}$ is found as :

$$
\begin{aligned}
\gamma_{i}-\gamma_{j} & =k\left(R_{i}-R_{j}\right)=-k \sin (\theta)\left[\left(\rho_{i}-\cos \left(\phi-\phi_{i}\right)\right)\right. \\
& \left.-\left(\rho_{j}-\cos \left(\phi-\phi_{j}\right)\right)\right] .
\end{aligned}
$$

Thus, the available power at point $\mathrm{M}$ would be: 


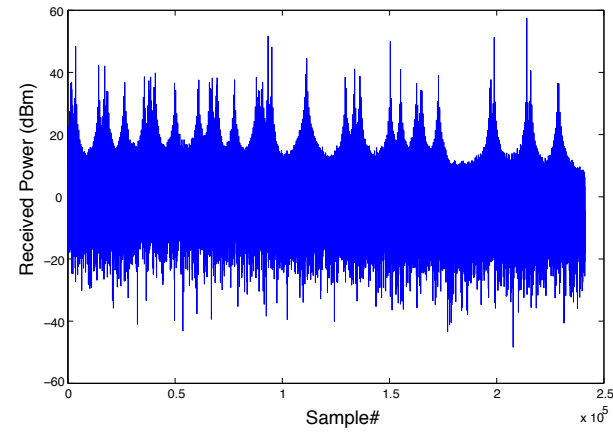

(a)

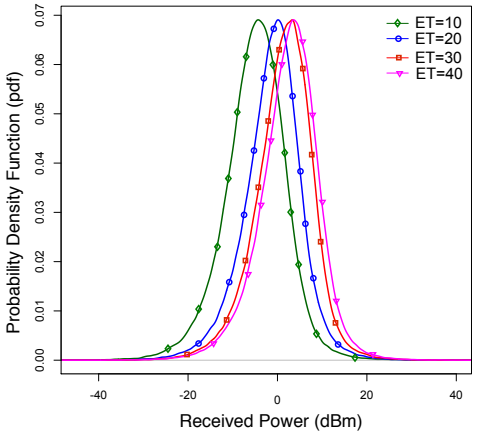

(b)

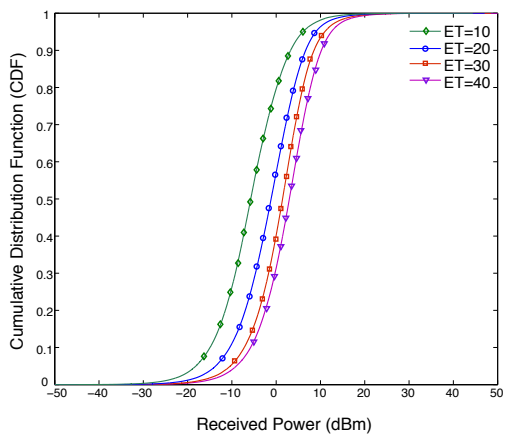

(c)

Fig. 3: (a) Received power samples over the WSN space with 40 ETs. (b) Log-Normal distributions of the network wide received power from multiple ETs. (c) Power outage probability based on CDF of the received power from multiple ETs.

$$
\begin{aligned}
P_{T}^{r} & =\sin ^{2}(\theta) P_{t} G_{t} G_{r}\left(\frac{\lambda}{4 \pi}\right)^{2}\left[\sum_{i=1}^{N} \frac{1}{\left(r-\rho_{i} \sin (\theta) \cos \left(\phi-\phi_{i}\right)\right)^{2}}\right. \\
+ & \left.\sum_{\substack{i=1 \\
i \neq j}}^{N} \sum_{j=1}^{N} \frac{\cos \left(\gamma_{i}-\gamma_{j}\right)}{\left(r-\rho_{i} \sin (\theta) \cos \left(\phi-\phi_{i}\right)\right)\left(r-\rho_{j} \sin (\theta) \cos \left(\phi-\phi_{j}\right)\right)}\right],
\end{aligned}
$$

$P_{T}^{r}=G_{r} \frac{\lambda^{2}}{4 \pi} S_{T}=G_{r} \frac{\lambda^{2}}{4 \pi}\left[\sum_{i=1}^{N} S_{i}+\sum_{\substack{i=1 \\ i \neq j}}^{N} \sum_{j=1}^{N}\left(\sqrt{S_{i} S_{j}} \cos \left(\gamma_{i}-\gamma_{j}\right)\right)\right]$

$$
\begin{aligned}
P_{T}^{r} & =\sin ^{2}(\theta) G_{r}\left(\frac{\lambda}{4 \pi}\right)^{2}\left[\sum_{i=1}^{N} \frac{P_{i} G_{i}}{R_{i}^{2}}\right. \\
& \left.\left.+\sum_{\substack{i=1 \\
i \neq j}}^{N} \sum_{j=1}^{N} \frac{\sqrt{G_{i} G_{j} P_{i} P_{j}}}{R_{i} R_{j}} \cos \left(\gamma_{i}-\gamma_{j}\right)\right)\right],
\end{aligned}
$$

where $P_{i}, P_{j}$, and $G_{r}$ are the transmission power of $E T_{i}$, the transmission power of $E T_{j}$, and the receiver gain, respectively. If ETs have the same antenna and transmission powers, then the available power at the receiver node can be simplified to

$P_{T}^{r}=\sin ^{2}(\theta) P_{t} G_{t} G_{r}\left(\frac{\lambda}{4 \pi}\right)^{2}\left[\sum_{i=1}^{N} \frac{1}{R_{i}^{2}}+\sum_{\substack{i=1 \\ i \neq j}}^{N} \sum_{j=1}^{N} \frac{\cos \left(\gamma_{i}-\gamma_{j}\right)}{R_{i} R_{j}}\right]$,

which is equal to Equation (2). This can be written in matrix form as: $P_{T}^{r}=(P)($ corr $)\left(R^{-1}\right)$ where the distance matrix $R^{-1}$ is $\left[1 / R_{1}, 1 / R_{2}, \ldots, 1 / R_{n}\right]$, the row vector $P$ is equal to $\left(\sin ^{2}(\theta) P_{t} G_{t} G_{r}\left(\frac{\lambda}{4 \pi}\right)^{2}\right) \cdot \mathbb{1}^{T}$, and the correlation matrix corr would be:

$$
\operatorname{corr}(i, j)=\cos \left(\gamma_{i}-\gamma_{j}\right) / R_{i}
$$

Finally, the amount of harvested power by the RF energy harvesting receiver would be:

$$
P_{H}=\eta P_{T}^{r}
$$

where $\eta$ is the RF-to-DC conversion efficiency. Also, the harvested voltage could be found by $V_{H}=F\left(P_{T}^{r}\right)$ where function $F$, similar to $2 \mathrm{D}$ case, depends on the energy harvesting circuit.

\section{Analysis and Simulation Results}

In this section, we analyze the performance of wireless energy transfer over a sensor network with multiple ETs. A variable number of ETs is randomly deployed in a $50 \times 50 \mathrm{~m}^{2}$ grid. Each ET transfers RF energy at center frequency $915 \mathrm{MHz}$ with Effective Isotropic Radiated Power (EIRP) equal to $4 \mathrm{~W}$, which is the maximum transmission power allowed by the Federal Communications Commission (FCC) for omni-directional energy transfer. The transmission parameters of all ETs are assumed to be identical. The parameters of the energy harvesting receiver are set according to our dual-stage energy harvester [9] with linear antenna gain of $6 \mathrm{dBi}$. The energy transfer of multiple ETs has been analyzed over the whole WSN. In particular, each time we have computed the received and harvested power in all points (samples) in the WSN deployment space, and accordingly found the network distributions of multiple wireless energy transfer. To this end, all sample points from the mesh grid range $[0,50]$ are selected with interval 0.1 . We assume two energy transfer scenarios. In the first scenario all ETs transmit omni-directional RF power at the same frequency, while in the second scenario (i.e., FDMAlike) each ET transmits power at a different frequency. This latter scenario is used as the base case for multiple energy transfer without interference. We focus our analysis on studying network-wide performance of concurrent wireless energy transfer in terms of power outage probability, energy 


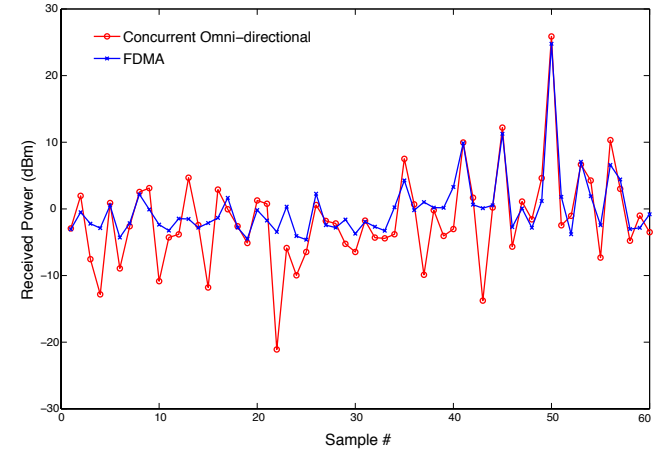

(a)

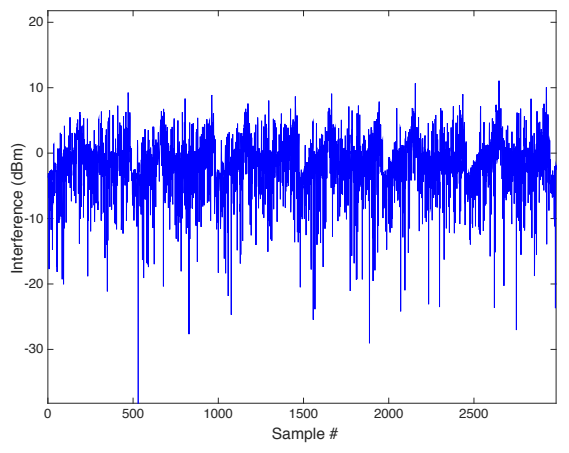

(b)

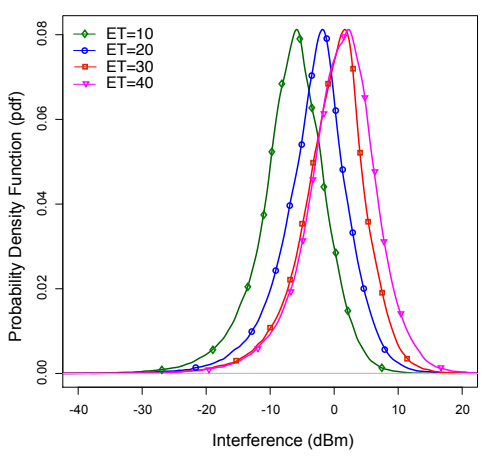

(c)

Fig. 4: (a) Comparison of received power with energy interference and without interference. (b) Energy interference samples over the sensor network with 40 ETs. (c) Log-Normal distributions of energy interference for multiple ETs.

interference, and harvested power and voltage. We have used Kolmogorov-Smirnov and Anderson-Darling tests to fit the distributions.

\section{A. Power outage probability}

First, we investigate the power outage probability in sensor network when ETs are transmitting omni-directional RF signals. The power outage probability is defined as the probability that the received power from multiple ETs at a destination in the network be less than a given threshold. Fig. 3a shows the received power over different samples in a network with 40 ETs. We see that the constructive and destructive interference of energy waves lead to different values of received energy at each destination. Figures $3 b$ and $3 \mathrm{c}$ depict that the PDF and CDF distributions of received power for different number of ETs, namely, 10, 20, 30 and 40. Fig. 3b shows that the received power over the network has Log-Normal distribution (Gaussian distribution in a logarithmic scale such as $\mathrm{dBm}$ ). Also, Fig. 3c indicates the effects of increasing ETs on the the received power over the network. Interestingly, the probability that the received power from multiple ETs becomes larger than $0 \mathrm{dBm}$ is $20 \%$ for $10 \mathrm{ETs}, 40 \%$ for $20 \mathrm{ETs}, 60 \%$ for $30 \mathrm{ETs}$, and $70 \%$ for 40 ETs. In addition, Fig. $3 \mathrm{c}$ represents the outage probability when multiple ETs transmitting at the same frequency. For example, when the minimum desired energy to power a sensor node is set to $-5 \mathrm{dBm}$, then the power outage probability is $50 \%$ for 10 ETs, $30 \%$ for 20 ETs, $15 \%$ for 30 ETs, and $10 \%$ for 40 ETs. Furthermore, the outage probability does not exhibit linear dependency with the number of ETs. Particularly, as the number of ETs increases, the rate at which the energy received changes does not increase at the same rate.

\section{B. Energy interference}

Next, we study the distributions of energy interference due to concurrent energy transfer from multiple ETs. To this end, we use FDMA transfer scheme as the base case for our comparisons, in which ETs transmit power at different frequencies and there is no interference between simultaneous energy waves. The different between the received energy from ETs in the same band and ETs transmitting with FDMA scheme is considered as the energy interference. Fig. 4a compares the received power over the network with 40 ETs. It is shown that the received power in the multienergy transfer at the same frequency could be at times greater, and at other times, smaller than FDMA. In fact, at some points over network, we see higher energy while other points receive less energy than FDMA. Furthermore, Fig. $4 \mathrm{~b}$ shows interference in a network with 40 ETs. We see that energy interference varies over the networks from locations with very high interference to ones with near zero interference. Finally, Fig. 4c depicts the distribution of energy interference for different number of ETs 10, 20, 30 , and 40. Interestingly, it is shown that energy interference over network has Log-Normal distribution (Gaussian distribution in $\mathrm{dBm}$ scale).

\section{Harvested voltage and power}

We next investigate the distributions of the harvested power and voltage over the sensor network. As shown in our previous work [9], regardless of the type of energy harvesting circuit, the output voltage vs. input power $(\mathrm{dBm})$ plot has an exponential curve due to use of either diodes or transistors in voltage multiplier sections of the energy harvesting circuit. Moreover, the efficiency curve (i.e. output power in $\mathrm{dBm}$ vs. input power in $\mathrm{dBm}$ ) has polynomial distribution. In this section, without loss of generality we emulate a dual-stage circuit which adaptively switches in low-power and high-power regions based on our energy harvesting circuit prototype presented in [9].

Figures $5 \mathrm{a}$ and $5 \mathrm{~b}$ show the PDF of the harvested power and harvested voltage over the whole sensor network for 10, 20, 30, and 40 ETs. Importantly, it is shown that harvested voltage over network has Rayleigh distribution, and harvested power clearly shows a dual-stage behavior in its distribution. Fig. 5c shows the CDF of harvested 


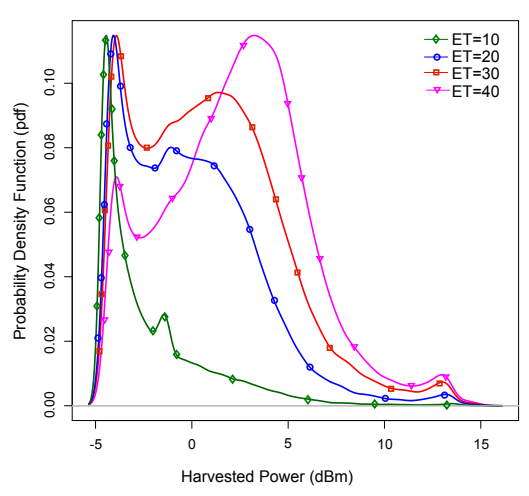

(a)

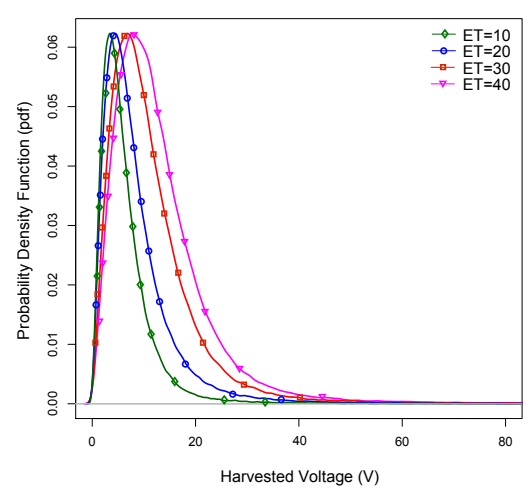

(b)

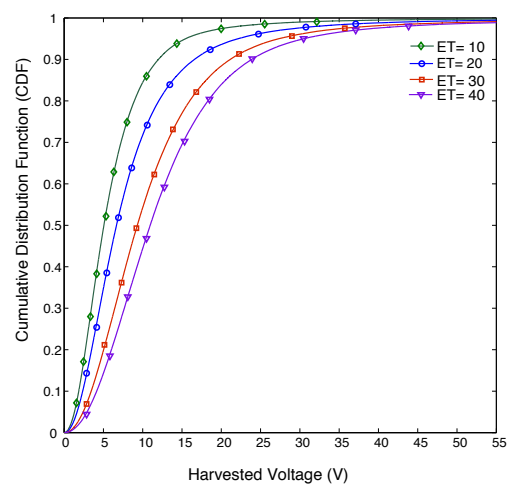

(c)

Fig. 5: (a) Dual-stage distributions of the harvested power $(\mathrm{dBm})$ over the sensor network with different number of ETs. (b) Rayleigh distributions of the harvested voltage over the network. (c) CDFs of the harvested voltage over the network.

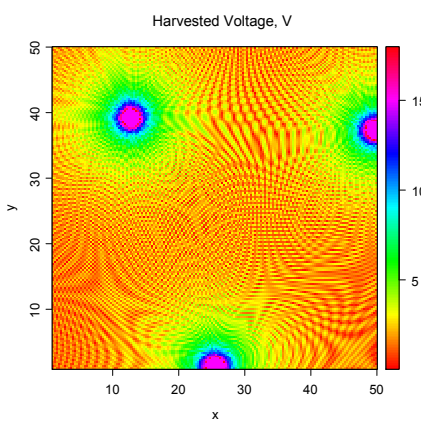

(a) 3 energy sources

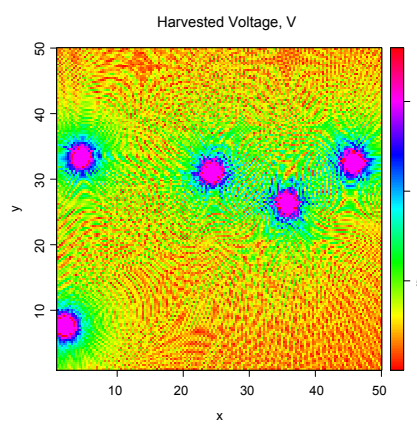

(b) 5 energy sources
Fig. 6: Intensity and patterns of harvested voltage.

voltage in the network. We see that the probability that the harvested voltage is less than $5 \mathrm{~V}$ is $50 \%$ for $10 \mathrm{ETs}$, $25 \%$ for 20 ETs, $20 \%$ for 30 ETs, and $15 \%$ for 40 ETs. Finally, Fig. 6 shows the harvested voltage map for 3 and 5 randomly deployed ETs, when the dual-stage harvesting circuit is used. The circles, which also have the highest harvested voltages, indicate the location of actual ETs. This figure depicts the randomness of energy patterns over the network, and shows as the number of ETs increases the randomness of energy patterns increases too. It can be observed even in points close to ETs such as $(14,10)$ in Fig. $6 \mathrm{a}$ and $(33,20)$ in Fig. $6 \mathrm{~b}$ voltage drops due to the destructive interference, while instead there are points at distance of ETs where show high harvested voltage owing to the constructive interference.

\section{CONCLUSIONS}

In this paper we derived closed matrix form expressions for the total harvestable power at any location in a WSN with multiple ETs by capturing the spatial correlation between ETs and their constructive and destructive energy interferences. We provided the formulas for plane and
3D WSN deployments. We analyzed the wireless energy over the network and presented the distributions of the received power, energy interference, and harvested voltage. Our results show that the received power from multiple ETs over the network and the network energy interference have Log-Normal distributions. We further observed that the harvested voltage over the network has a Rayleigh distribution.

\section{ACKNOWLEDGMENTS}

This work was supported in part by the US National Science Foundation under research grant CNS-1143681.

\section{REFERENCES}

[1] S. Basagni, M. Y. Naderi, C. Petrioli, and D. Spenza, "Wireless sensor networks with energy harvesting," in Mobile Ad Hoc Networking: Cutting Edge Directions, S. Basagni, M. Conti, S. Giordano, and I. Stojmenovic, Eds. Hoboken, NJ: John Wiley \& Sons, Inc., March 5 2013, ch. 20, pp. 703-736.

[2] M. Naderi, K. R. Chowdhury, S. Basagni, W. Heinzelman, S. De, and S. Jana, "Experimental study of concurrent data and wireless energy transfer for sensor networks," in Proceedings of IEEE GLOBECOM, 2014.

[3] J. Kraus and D. Fleisch, Electromagnetics: With Applications, 5th Ed. WCB/McGraw-Hill, 1999.

[4] B. Sklar, Digital communications: Fundamentals and applications, 2nd ed. Prentice-Hall PTR, 2001.

[5] C. Balanis, Antenna Theory: Analysis and Design. Wiley, 2012.

[6] H. Ochiai, P. Mitran, H. Poor, and V. Tarokh, "Collaborative beamforming for distributed wireless ad hoc sensor networks," IEEE Transactions on Signal Processing, vol. 53, no. 11, pp. 4110-4124, November 2005.

[7] R. Harrington, "Sidelobe reduction by nonuniform element spacing," IEEE Transactions on Antennas and Propagation, vol. 9, no. 2, pp. 187-192, March 1961.

[8] P. Lorrain, D. Corson, and F. Lorrain, Electromagnetic Fields and Waves: Including Electric Circuits. Freeman, 1988.

[9] P. Nintanavongsa, U. Muncuk, D. Lewis, and K. Chowdhury, "Design optimization and implementation for RF energy harvesting circuits," IEEE Journal on Emerging and Selected Topics in Circuits and Systems, vol. 2, no. 1, pp. 24-33, March 2012. 